\title{
Comportamentos e práticas sexuais de homens que fazem sexo com homens
}

\author{
Sexual behaviors and practices of men who have sex with men
}

Conductas y prácticas sexuales de los hombres que tienen sexo con hombres

\section{Diego Jorge Maia Lima', Priscila Fontenele de Paula', Priscila de Souza Aquino', Paula Renata Amorim Lessa', Maria Leonor Costa de Moraes", Denise de Fátima Fernandes Cunha', Ana Karina Bezerra Pinheiro'}

' Universidade Federal do Ceará, Centro de Ciências da Saúde, Programa de Pós-Graduação em Enfermagem. Fortaleza-CE, Brasil.

" Universidade de Brasília, Programa de Pós-Graduação em Enfermagem. Brasília-DF, Brasil.

\author{
Submissão: 29-01-2013Ａprovação: 10-09-2014
}

\section{RESUMO}

Objetivou-se identificar comportamentos e práticas sexuais de homens que fazem sexo com homens no contexto da vulnerabilidade ao HIV/AIDS. Estudo transversal, exploratório descritivo. Foi realizado em um local de sociabilidade gay de Fortaleza, no Estado do Ceará, entre novembro de 2010 e março de 2011, por meio de entrevista com 189 homens que fazem sexo com homens. Encontrou-se uma amostra composta, majoritariamente, por jovens, solteiros e com alto nível educacional. A história sexual demonstrou o início precoce da vida sexual, com prevalência elevada de relação sexual com parceira do sexo oposto. Houve alta frequência de testagem para o HIV. As práticas sexuais revelaram prevalência superior da realização de sexo oral e anal, bem como altos níveis de proteção no sexo anal, apesar de baixa no sexo oral. Constatou-se uma maior incorporação das práticas de prevenção em relação ao panorama nacional do início da epidemia.

Descritores: Comportamento Sexual; Homossexualidade Masculina; Fatores de Risco; Vulnerabilidade em Saúde; Enfermagem.

\section{ABSTRACT}

The objective was to identify behaviors and sexual practices of men who have sexual relations with other men in the context of vulnerability to HIV/AIDS. This was a cross-sectional, exploratory and descriptive study. It was carried out in a gay sociability place in Fortaleza, Ceará, Brazil, between November 2010 and March 2011, through interviews with 189 men who have sex with men. The ethical aspects were respected. We found a sample consisting mostly by young, single, and highly educated men. The sexual history demonstrated the early onset of sexual activity, with a high prevalence of sexual intercourse with a partner of the opposite sex. There was also a high prevalence of HIV testing. Sexual practices revealed high prevalence of performing oral and anal sex, as well as high levels of protection in anal sex, despite the low protection in oral sex. A greater incorporation of prevention practices was found compared to the national scene in the beginning of the disease outbreak.

Key words: Sexual Behavior; Homosexuality Male; Risk Factors; Health Vulnerability; Nursing.

\section{RESUMEN}

El objetivo fue identificar los comportamientos y las prácticas sexuales de los hombres que tienen sexo con hombres en el contexto de la vulnerabilidad al VIH/SIDA. Fue un estudio transversal, descriptivo y exploratorio. Se celebró en una sociabilidad local gay de Fortaleza, Ceará, Brasil, entre noviembre de 2010 y marzo de 2011, a través de entrevistas con 189 hombres que tienen sexo con hombres. Se encontró una muestra compuesta en su mayoría por jóvenes, solteros y con alto nivel de educación. La historia sexual demostró el inicio temprano de la actividad sexual, la alta prevalencia de relaciones sexuales con una pareja del sexo opuesto. Hubo alta prevalencia de la prueba del VIH. Las prácticas sexuales revelaron una alta prevalencia de realizar sexo oral y anal, así como altos niveles de protección en el sexo anal, a pesar de la baja protección en el sexo oral. Se encontró una mayor incorporación de las prácticas de prevención en relación con la escena nacional en el inicio de la epidemia.

Palabras clave: Conducta Sexual; Homosexualidad Masculina; Factores de Riesgo; Vulnerabilidad en Salud; Enfermería. 


\section{INTRODUÇÃO}

No contexto epidemiológico da AIDS, a população de homens que fazem sexo com homens (HSH) é considerada uma das mais vulneráveis, apresentando um elevado número de casos dessa doença nas categorias de exposição sexual, homo e bissexual, apesar de demonstrar uma tendência à estabilização nos últimos anos no Brasil. Tais dados epidemiológicos apontam para a prevalência preocupante de $39,4 \%$ de casos da doença decorrentes de exposição sexual nessas categorias ${ }^{(1)}$.

Nesse cenário, a categoria HSH vem sendo amplamente utilizada para designar homossexuais, bissexuais e outros homens que assumem tal prática, mas que podem sentir dificuldade em se definirem como homossexuais. Essa terminologia tem sido adotada por grande parte dos estudos de saúde coletiva, que concentram esforços para a compreensão da dinâmica da epidemia de AIDS nessa população(2).

Esses inquéritos têm investigado os diversos fatores que favorecem a transmissão do HIV/AIDS no subgrupo de HSH, como a adoção de práticas sexuais desprotegidas, a aquisição de comportamentos de risco, e o preconceito e a discriminação, o que torna os HSH uma das populações mais vulneráveis no contexto da epidemia do HIV/AIDS ${ }^{(2-3)}$.

Ao longo da epidemia, o conceito de risco esteve atrelado às políticas e práticas preventivas direcionadas a populações específicas, muitas vezes com conotação discriminatória e estigmatizante. Dessa forma, atualmente o conceito de "vulnerabilidade" é adotado, pois abrange a percepção do adoecimento como resultado de um conjunto de aspectos, não apenas individuais, mas também coletivos e contextuais, que acarretam maior suscetibilidade ao sujeito ${ }^{(4)}$.

Cabe ressaltar que o enfrentamento da epidemia de AIDS em qualquer segmento populacional, incluindo a população $\mathrm{HSH}$, exige o reconhecimento da vulnerabilidade, especialmente de aspectos relacionados aos comportamentos e práticas sexuais adotadas pela população citada. Desse modo, é imprescindível a realização de estudos que investiguem as práticas sexuais na população específica de $\mathrm{HSH}$, com vistas ao desenvolvimento de intervenções direcionadas ao subgrupo e à avaliação das iniciativas já existentes para prevenção do HIV/AIDS entre HSH.

O presente estudo objetivou identificar os comportamentos e as práticas sexuais de $\mathrm{HSH}$, a fim de reconhecer o contexto de vulnerabilidade em que a referida população está inserida, bem como identificar o perfil social e sexual dos $\mathrm{HSH}$.

Nesse contexto, o enfermeiro, como um dos principais promotores de saúde, detém uma grande responsabilidade, desempenhando esse papel em sua prática profissional por meio de ações específicas para esse grupo, como a realização de estratégias educativas, a participação em programas de prevenção e no acolhimento humanizado, por meio de uma escuta ativa das demandas dos HSH nas diversas instituições da saúde.

\section{METODOLOGIA}

Tratou-se de um estudo transversal do tipo exploratório descritivo. $\mathrm{O}$ inquérito foi realizado em uma boate localizada no centro da cidade de Fortaleza, no Estado do Ceará, reconhecida como um dos principais locais de sociabilidade gay da cidade. O local foi descrito minuciosamente em estudo etnográfico, sendo evidenciado como um local tradicional de sociabilidade dessa população, o que justificou a seleção do mesmo para realização desta pesquisa ${ }^{(5)}$. A opção pelo recrutamento da amostra em locais de sociabilidade gay seguiu uma tendência observada em outros estudos realizados com a referida população ${ }^{(2,6)}$.

Empregou-se a amostragem não probabilística, método utilizado em situações que não é possível construir uma amostra-padrão adequada de locais ou situações em que integrantes de uma subpopulação se reúnem. Esse tipo de amostra gera dados relevantes e vem sendo utilizada nos estudos direcionados à população $\mathrm{HSH}^{(6-7)}$.

O tamanho amostral foi calculado por meio da fórmula para populações finitas, totalizando $189 \mathrm{HSH}$, que atenderam aos os seguintes critérios de inclusão: maiores de 18 anos, brasileiros, que tivessem se relacionado sexualmente pelo menos uma vez no último ano e que frequentassem locais de sociabilidade gay.

A coleta de dados ocorreu no período de novembro de 2010 a março de 2011. A abordagem dos sujeitos deu-se de maneira aleatória antes da entrada no local supracitado. $\mathrm{O}$ formulário foi preenchido com dados sociodemográficos, de história e de práticas sexuais. Esse instrumento foi construído a partir da adaptação de instrumento utilizado em outro estudo de âmbito nacional(2).

A análise dos dados consistiu na descrição das frequências e médias, dispostas na forma de tabelas ilustrativas. O programa Statistical Package for the Social Sciences (SPSS), versão 17.0, foi utilizado e discutiram-se os resultados conforme a literatura pertinente. Os aspectos éticos e legais envolvendo a pesquisa com seres humanos foram respeitados. A pesquisa foi aprovada pelo Comitê de Ética em Pesquisa da Universidade Federal do Ceará, conforme protocolo 247/10.

\section{RESULTADOS}

Os dados referentes às características sociodemográficas da amostra estão disponibilizados na Tabela 1.

Tabela 1 - Distribuição dos dados sociodemográficos de homens que fazem sexo com homens-HSH, Fortaleza, Ceará, 2011

\begin{tabular}{ll}
\hline Caracterização & $\boldsymbol{f ( \% )}$ \\
\hline $\begin{array}{l}\text { Faixa etária, anos }(\mathrm{n}=189) \\
18-25\end{array}$ & $95(50,3)$ \\
$25-34$ & $68(35,8)$ \\
$35-44$ & $21(11,0)$ \\
$45-55$ & $5(2,6)$ \\
Escolaridade $(\mathrm{n}=189)$ & \\
Fundamental incompleto & $3(1,6)$ \\
Fundamental completo & $4(2,1)$ \\
Médio incompleto & $17(9,0)$ \\
\hline
\end{tabular}


Tabela 1 (cont.)

\begin{tabular}{ll}
\hline Caracterização & $\boldsymbol{f ( \% )}$ \\
\hline Escolaridade ( $\mathrm{n}=199)$ & \\
Médio completo & $89(47,1)$ \\
Superior incompleto & $35(18,5)$ \\
Superior completo & $28(14,8)$ \\
Pós-Graduação & $13(6,9)$ \\
Estado civil (n=199) & \\
Solteiro & $162(85,7)$ \\
Casado/amigado com homem & $24(12,7)$ \\
Separado & $1(0,5)$ \\
Divorciado & $2(1,1)$ \\
\hline
\end{tabular}

Com relação à idade, todos os participantes eram maiores de 18 anos, com média de idade 26,54 anos, sendo a mínima de 18 e a máxima de 55 anos, com desvio padrão de 7,40. A escolaridade dos sujeitos, medida por nível de escolaridade, foi considerada alta, pois $87,3 \%$ (155) dos sujeitos estudados eram homens com, no mínimo, Ensino Médio completo.

\section{História sexual}

Os dados referentes ao comportamento sexual dos participantes, como sexarca, relação heterossexual, história de Doenças Sexualmente Transmissíveis (DST) e realização de teste anti-HIV estão dispostos na Tabela 2.

Tabela 2 - Distribuição dos dados da história sexual de homens que fazem sexo com homens, Fortaleza, Ceará, 2011

\begin{tabular}{ll}
\hline História sexual & \multicolumn{1}{c}{$(\mathbf{\%})$} \\
\hline $\begin{array}{l}\text { Idade da primeira relação sexual, anos }(\mathrm{n}=189) \\
\quad \text { Menos de } 10\end{array}$ & $18(9,6)$ \\
$11-15$ & $99(52,3)$ \\
$16-20$ & $64(33,8)$ \\
$21-23$ & $7(3,7)$ \\
Não lembra & $1(0,5)$ \\
Relação heterossexual $(\mathrm{n}=189)$ & \\
Sim & $117(61,9)$ \\
Não & $72(38,1)$ \\
História pessoal de DST $(\mathrm{n}=189)$ & \\
Sim & $26(13,8)$ \\
Não & $162(85,7)$ \\
Não lembra & $1(0,5)$ \\
Tratamento de DST $(\mathrm{n}=26)$ & \\
Sim & $21(86,2)$ \\
Não & $5(11,1)$ \\
Realização do teste anti-HIV $(\mathrm{n}=189)$ & $139(73,5)$ \\
Sim & $50(26,5)$ \\
Não &
\end{tabular}

DST: Doenças Sexualmente Transmissíveis.
Referente à sexarca, a idade média de início sexual foi de 14,6 anos, sendo a menor 6 anos e a maior 23 anos, com desvio padrão de 3,1 .

\section{Práticas sexuais}

As práticas sexuais também foram investigadas, e os dados relativos a essas informações estão na Tabela 3.

Tabela 3 - Distribuição das práticas sexuais de homens que fazem sexo com homens, Fortaleza, Ceará, 2011

\begin{tabular}{ll}
\hline Práticas sexuais & $\boldsymbol{f ( \% )}$ \\
\hline Sexo oral ( $\mathrm{n}=189)$ & $182(96,3)$ \\
Sim & $7(3,7)$ \\
Não & \\
Uso do preservativo no sexo oral $(\mathrm{n}=182)$ & $60(31,7)$ \\
$\quad$ Sempre & $42(22,2)$ \\
Às vezes & $77(40,7)$ \\
Não usa & $3(1,6)$ \\
Apenas quando realizo (insertivo) & \\
Sexo anal ( $\mathrm{n}=189)$ & $185(97,9)$ \\
Sim & $4(2,1)$ \\
Não & \\
Uso do preservativo no sexo anal (n=185) & $139(73,5)$ \\
Sempre, independente do tipo & $2(1,1)$ \\
Apenas quando é passivo (receptivo) & $7(3,7)$ \\
Apenas quando é ativo (insertivo) & $24(12,7)$ \\
Não usa & $13(6,9)$ \\
Às vezes & \\
\hline
\end{tabular}

A maioria dos respondentes $(182 ; 96,3 \%)$ relatou a realização de sexo oral. Quanto à prática do sexo anal, também quase totalidade $(185 ; 97,8 \%)$ dos sujeitos a referiu. Foram encontrados resultados que ressaltam a elevada prevalência da prática do sexo anal desprotegido na amostra estudada, pois 12,7\% (24) dos entrevistados referiram que não usavam proteção e $6,9 \%$ (13) assumiram a utilização da proteção "às vezes".

\section{DISCUSSÃO}

A idade dos participantes do estudo denotou uma população jovem usuária dos locais de sociabilidade gay, congruente com o apresentado em estudos semelhantes. Pesquisa desenvolvida com amostragem semelhante, na qual entrevistaram-se $465 \mathrm{HSH}$ na cidade de Brasília, revelou amostra formada predominantemente por jovens, com 92,7\% entre 18 e 39 anos e média de 27 anos de idade, corroborando os dados do presente estudo ${ }^{(7)}$.

Os estudos realizados com essa população têm demonstrado, de maneira geral, um nível de escolaridade elevado, o que está em consonância com o presente inquérito. Pesquisa realizada no Rio de Janeiro demonstrou que 44\% (176) dos entrevistados $(400 \mathrm{HSH})$ informaram ter concluído o Ensino Médio, 29,5\% (118) o Superior incompleto e 102 (25,5\%) o 
Superior completo. Logo, 55\% possuíam o Ensino Superior incompleto ou mais ${ }^{(6)}$.

No tocante ao estado conjugal, os achados da presente pesquisa estiveram de acordo com a literatura(2). O estado civil solteiro foi referido por $68,7 \%$ dos $\mathrm{HSH}$ de inquérito nacional realizado com $487 \mathrm{HSH}^{(2)}$.

Em relação à iniciação sexual precoce, a idade média de início de relacionamento sexual de $17 \%$ dos entrevistados em Campo Grande esteve por volta dos 8 anos de idade; $44 \%$ estiveram em torno dos 13 anos de idade, caracterizando um importante elemento favorável à aquisição de DST, inclusive a $\operatorname{AIDS}^{(8)}$. O início sexual precoce está associado ao não uso ou uso inadequado do preservativo, além do uso do tabaco, álcool e consumo de outras drogas ${ }^{(9)}$.

Percebeu-se elevada prevalência de HSH que referiram relação sexual com mulheres em algum momento da vida. Tal fato pode ter relação com o período em que a identidade sexual ainda não é totalmente compreendida pelo homossexual, levando-o à busca de diversas experiências sexuais, inclusive a relação heterossexual ${ }^{(10)}$. Por outro lado, pode-se pensar nesse achado como uma expressão da bissexualidade. Estudo evidenciou que a vulnerabilidade para HIV/AIDS foi mais frequente entre os homens que relataram atividade bissexual. Os comportamentos sexuais e de proteção diferem conforme gênero e estabilidade da parceria, havendo maior desproteção nas parcerias fixas do gênero feminino entre os bissexuais ${ }^{(11)}$.

Ademais, pensa-se também na necessidade de, muitas vezes, esconder a orientação sexual, pois o homossexual pode ser vítima de preconceitos ou isolamento da sociedade e, inclusive, da própria família. Estudo sobre o preconceito contra homossexuais aponta para presença de um esquema social discriminatório contra o homossexual na sociedade brasileira ${ }^{(12)}$.

Nota-se que, embora o número de homens que afirmaram história pessoal de DST não seja o mais prevalente, esse dado ainda é significativo. Em pesquisa desenvolvida no Distrito Federal, tendo a participação de 465 homens, 7,5\% afirmaram ter apresentado algum sintoma de DST nos últimos 6 meses $^{(7)}$. Já no presente estudo foi considerável o número de pessoas com história pessoal de DST e, embora não tenha sido a maior prevalência, os números demonstraram a vulnerabilidade desse grupo frente à aquisição dessas doenças.

Associada à vulnerabilidade para DST, encontrou-se, também, alta prevalência de realização de testagem anti-HIV. Nesse sentido, percebe-se uma consonância com investigação na cidade do Rio de Janeiro, na qual 72,5\% dos entrevistados comunicaram a realização do teste anti-HIV(6). A alta prevalência de testagem entre a população estudada pode estar relacionada com a prática recorrente de comportamentos de risco, que provocaria medo de ter contraído o HIV e que levaria à procura pelo teste, em parte como forma de reduzir a culpa e o medo relacionados à prática insegura, além da falsa segurança proporcionada por um resultado negativo ${ }^{(7)}$.

A investigação acerca do sexo oral esteve em consonância com outras pesquisas. A preferência pelo sexo oral foi investigada em uma pesquisa com $577 \mathrm{HSH}$, usuários da internet, na qual se aplicou uma escala de gradação de prazer em relação ao sexo oral insertivo e receptivo. Assim, foi considerada uma prática "prazerosa" por 74,2\% e 73,9\% dos entrevistados, respectivamente. Existem evidências científicas que apontam para o sexo oral como uma via de transmissão para o HIV. Porém, os estudos realizados até o momento não são precisos quanto à quantificação e à qualificação desse risco ${ }^{(13)}$.

Cabe notar a grande preferência da população de $\mathrm{HSH}$ estudada pela realização da prática do sexo anal. Percebe-se que a realização desse tipo de prática pelos $\mathrm{HSH}$ tem, na realização dessa prática, uma importante dimensão da identidade sexual, possuindo um significado simbólico dentro do cenário das práticas sexuais dessa população ${ }^{(14)}$. De forma semelhante, entre as práticas sexuais mais comuns dos $\mathrm{HSH}$ entrevistados na Região Sul do país, destacou-se o sexo anal insertivo com $87 \%$ e o sexo anal receptivo com $73 \%{ }^{(15)}$. Sabe-se que o sexo anal insertivo e o receptivo são consideradas como práticas de grande risco para a infecção pelo HIV/AIDS, dentro de uma hierarquia de prevenção ${ }^{(2)}$.

\section{CONSIDERAÇÕES FINAIS}

Os achados desta pesquisa permitiram constatar que, apesar de não existirem estratégias de ampla escala no contexto da prevenção da transmissão do HIV/AIDS entre os $\mathrm{HSH}$, verificaram-se mudanças que apontaram para uma maior proteção dentro desse grupo.

Notou-se que, apesar do uso do preservativo no sexo anal estar presente na maior parte da amostra, cabe ressaltar ainda a existência de vulnerabilidades pela prática desprotegida. Em contrapartida, os dados epidemiológicos vêm apontando para uma estabilização do número de casos diagnosticados, por meio da contaminação homossexual e bissexual. Tais resultados apontaram para o crescimento da capacidade desse grupo em reagir positivamente aos desafios impostos pela epidemia.

Contudo, a suscetibilidade de determinados grupos e a identificação e compreensão de suas particularidades constituem, ainda, um grande desafio a ser enfrentado em todos os espaços em que condições de risco para aquisição de DST/ AIDS estejam presentes.

Ressalta-se a pouca adesão do preservativo na realização do sexo oral entre os sujeitos estudados. Sugere-se uma melhor compreensão dessa especificidade por meio de um conhecimento mais aprofundado, com o objetivo de subsidiar intervenções que visem à redução do comportamento de risco entre os $\mathrm{HSH}$. Considera-se importante a realização de novas pesquisas que objetivem aprofundar a investigação sobre as razões associadas a não utilização de preservativos, seja na prática do sexo oral ou anal.

Cabe salientar que, devido aos métodos de amostragem, os resultados obtidos não permitiram uma generalização para toda população de HSH, mas demonstraram informações importantes, que podem subsidiar a construção de ações educativas, de políticas e programas direcionados para esse segmento populacional.

Nesse contexto, o profissional enfermeiro deve desempenhar um papel fundamental na promoção da saúde sexual desse grupo específico, por meio de uma atenção humanizada pautada no cuidado holístico, livre de preconceitos, e em atividades de caráter educativo, com vistas a mudanças de comportamento, em busca da prática sexual segura. 


\section{REFERÊNCIAS}

1. Ministério da Saúde (BR). Boletim epidemiológico-Aids. Programa Nacional de DST e Aids. Brasília (DF): Ministério da Saúde; 2013.

2. Brignol S, Dourado I. Inquérito sociocomportamental sobre as práticas sexuais desprotegidas entre homens que fazem sexo com homens usuários da Internet. Rev Bras Epidemiol [Internet]. 2011 [acesso em 5 de setembro de 2014];14(3):423-34. Disponível em: http://www. scielo.br/scielo.php?script =sci_arttext\&pid = S1415$-790 \times 2011000300007$

3. Ministério da Saúde (BR). Política Nacional de Saúde Integral de Lésbicas, Gays, Bissexuais, Travestis e Transsexuais [Internet]. Brasília (DF): Ministério da Saúde; 2010 [acesso em 5 de setembro de 2014]. Disponível em: http://www. abglt.org.br/docs/PoliticaNacional_Saudelntegral_LGBT.pdf

4. Ayres JRC. O conceito de vulnerabilidade e as práticas de saúde: novas perspectivas e desafios. In: Czreresnia D, Freitas CM, organzadores. Promoção da saúde: conceitos, reflexões, tendências. Rio de Janeiro: Fiocruz; 2009. p. 121-43.

5. Coelho JFJ. Bastidores e estreias: performers trans e boates gays "abalando" a cidade [dissertação]. Fotaleza (CE): Universidade Federal do Ceará; 2009.

6. Raxachi JC, Terto Júnior V, Garcia J, Pimenta C, Almeida V, Parker R. Práticas sexuais e conscientização sobre AIDS: uma pesquisa sobre o comportamento homossexual e bissexual [Internet]. Rio de Janeiro: ABIA; 2007 [acesso em 5 de setembro de 2014]. Disponível em: http://www.abiaids.org.br/_img/media/colecao_abia_5internet.pdf

7. Lima FSS, Silva MJG, Godoi AMM, Mérchan-Hamann E. Homens que fazem sexo com homens: uso dos serviços para prevenção/controle de HIV/AIDS em Brasília-DF. Com Ciências Saúde [Internet]. 2008 [acesso em 5 de setembro de 2014];19(1):25-34. Disponível em: http://www.escs.edu. br/pesquisa/revista/2008Vol19_1art03homensquefazem.pdf
8. Andrade SMO, Tamaki EM, Vinha JM, Pompilio MA, Prieto $\mathrm{CW}$, Barros LM, et al. [Vulnerability of men who have sex with men in the context of AIDS]. Cad Saúde Pública. 2007;23(2):479-82. Portuguese.

9. World Health Organization (WHO). Health Police for Children and Adolescents. HBSC: Inequalites in young people's health. Health behavior in school-aged children. Scotland (UK): Child and Adolescent Health Research Unit (CAHRU); 2008. International Report from the 2005/2006.

10. Taquette SR, Vilhena MM, Santos UPP, Barros MMV. Relatos de experiência homossexual em adolescentes masculinos. Ciênc Saúde Coletiva. 2005;10(2):399-407.

11. Greco $M$, Silva $A P$, Merchan-Hamann $E$, Jeronymo $M L$, Andrade JC, Greco DB. Diferenças nas situações de risco para HIV de homens bissexuais em suas relações com homens e mulheres. Rev Saúde Pública. 2007;41(2):109-17.

12. Fleury ARD, Torres ARR. Análise psicossocial do preconceito contra homossexuais. Estud Psicol [Internet]. 2007 [acesso em 5 de setembro de 2014];24(4):475-86. Disponível em: http://www.scielo.br/pdf/estpsi/v24n4/ v24n4a07.pdf

13. Funari SL. Sexo oral e HIV entre homens que fazem sexo com homens. Cad Saúde Pública. 2003;19(6):1841-4.

14. Gondim RC, Kerr-Pontes LRS. Homo-bissexualidade masculina: um estudo sobre práticas sexuais desprotegidas em Fortaleza. Rev Bras Epidemiol [Internet]. 2000 [acesso em 5 de setembro de 2014];(3):1-3. Disponível em: http:// www.scielosp.org/pdf/rbepid/v3n1-3/05.pdf

15. Ferraz EA, Schwenck C, Quental $\mathrm{Cl}$. Homens que fazem sexo com homens: vulnerabilidades a partir de comportamentos sexuais. In: XIV Encontro Nacional de Estudos Populacionais; 2004 Set 20-24. Caxambu (MG), Brasil. Caxambu: ABEP-MG; 2004. p.17. 